\title{
Medicamentos sobrantes y caducados en el hogar ¿su almacenaje y desecho representan un problema de salud pública?
}

\author{
Leftover and expired medicines in households: Is their \\ storage and disposal a public health problem?
}

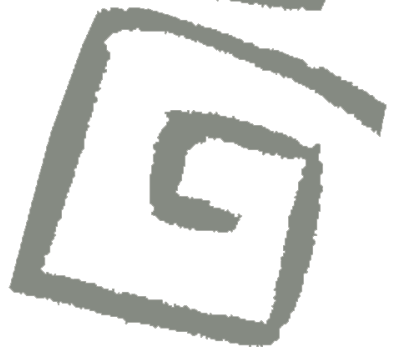

Juan Martín Calderón', Mónica Tarapués²

'Estudiante de Medicina. Facultad de Ciencias Médicas, Universidad Central del Ecuador, Quito, Ecuador. $₫$ iD

${ }^{2}$ Doctora en Farmacología. Docente, Facultad de Ciencias Médicas, Universidad Central del Ecuador, Quito, Ecuador. $\triangle$ iD
RESUMEN Los medicamentos sobrantes y caducados en el hogar deberían ser eliminados de una manera que garantice la seguridad de la población, y que tenga un impacto negativo mínimo en el medio ambiente. Desde esta perspectiva se desarrolló el presente estudio cuyo objetivo fue conocer las prácticas de desecho de medicamentos del botiquín familiar en estudiantes de medicina en Quito-Ecuador. Entre diciembre de 2018 y enero de 2019, se encuestaron 498 estudiantes de diferentes semestres, y se evidenció que hasta un 30,3\% de estudiantes alguna vez desechó los medicamentos a través del inodoro, y un 7,2\% reconoció que sacaba los medicamentos del empaque primario, para depositarlos en la basura común. Como objetivo secundario se analizaron los medicamentos caducados y sobrantes del botiquín familiar de los encuestados. Se encontró que los medicamentos más frecuentes fueron metformina, seguido por acetaminofén, espironolactona e ibuprofeno. El estudio muestra la necesidad de desarrollar estrategias multisectoriales para la implementación de políticas sobre el desecho doméstico, las cuales permitirán controlar, y en el mejor de los casos, disminuir el impacto negativo tanto ambiental como en salud pública.

PALABRAS CLAVES Residuos de Medicamentos; Ecotoxicología; Farmacovigilancia; Farmacoepidemiología; Almacenamiento de Medicamentos; Ecuador.

\begin{abstract}
Leftover and expired medicines in households must be disposed of in such a way as to ensure the population's safety, while generating the lowest possible negative impact on the environment. In this context, the aim of this study was to explore drug disposal practices related to home medicine cabinets among medical students in Quito, Ecuador. Between December 2018 and January 2019, 498 students from different semesters were surveyed. Data show that up to $30.3 \%$ of students had flushed their medicines down the toilet at least once, while $7.2 \%$ acknowledged that they had removed the medicines from their packaging to deposit them in a household garbage disposal. A secondary aim of the study was to analyze expired and leftover drugs in participants' medicine cabinets. Metformin was the most common medication found, followed by acetaminophen, spironolactone, and ibuprofen. This study shows the urgent need to develop multisectoral strategies for the implementation of policies on pharmaceutical domestic waste, which will make it possible to control and reduce the negative impact on both the environment and public health.
\end{abstract}

KEY WORDS Drug Residues; Ecotoxicology; Pharmacovigilance; Pharmacoepidemiology; Drug Storage; Ecuador. 


\section{INTRODUCCIÓN}

El botiquín familiar se caracteriza por proveer los elementos necesarios para tratar dolencias leves, y quizás incluir medicamentos de venta libre mientras se espera por asistencia médica ${ }^{(1,2)}$. Sin embargo, es frecuente que contenga medicamentos sobrantes de tratamientos previos o sencillamente otros medicamentos adquiridos. Esta problemática se hace más evidente cuando existen miembros de la familia con enfermedades crónicas y adultos mayores ${ }^{(3,4,5)}$; por lo que los botiquines familiares podrían albergar de manera injustificada medicamentos producto del cambio de tratamientos crónicos ${ }^{(6)}$.

Estudios tanto en América Latina como en otras regiones señalan que, en algunos de los medicamentos sobrantes almacenados en los hogares, es imposible identificar la fecha de caducidad porque carecen de sus envases secundarios (frascos o cajas). En otros casos, no se dispone de los prospectos o información para pacientes. Mientras que, en casos más extremos, los medicamentos están fuera de su envase primario (blíster) o dicho envase fue cortado y no se tiene información de su fecha de caducidad, convirtiéndolos en medicinas no aptas para el consumo ${ }^{(2,5,7)}$.

En este contexto, los medicamentos sobrantes o caducados deben ser eliminados de una manera que garantice la salud y la seguridad de la población, y que impliquen el mínimo impacto negativo en el medio ambiente. Estos medicamentos destinados a la disposición final toman el nombre de desechos farmacéuticos, y su eliminación adecuada está constituida por una serie de operaciones controladas para garantizar una destrucción segura ${ }^{(8,9,10)}$.

En países europeos se han desarrollado estrategias que permiten el desecho seguro de medicamentos. Mediante estas estrategias los medicamentos caducados y sobrantes de origen domiciliario reciben un tratamiento de eliminación específico y diferenciado ${ }^{(11)}$. Un ejemplo consiste en la estrategia española de Sistema Integrado de Gestión de Residuos y Envases (SIGRE). Los puntos de recolección del SIGRE se encuentran en todas las farmacias del país, permitiendo a los ciudadanos ser parte activa de la recolección de los medicamentos caducados o sobrantes ${ }^{(12,13)}$.

En los países de América Latina todavía se carece de estrategias nacionales para la eliminación segura de medicamentos caducados o sobrantes. En Ecuador, a partir del año 2017, la Empresa Pública Metropolitana de Aseo (EMASEO-EP), en coordinación con la Secretaría de Ambiente y la Empresa Pública Metropolitana de Gestión Integral de Residuos Sólidos (EMGIRS-EP) se encargó de coordinar e implementar la campaña "Quito a reciclar", un programa de recolección diferenciada que se aplica únicamente en la ciudad de Quito. Este programa consiste en recolectar de manera diferenciada residuos domésticos especiales y peligrosos tales como focos ahorradores, lacas y pinturas aerosol, y medicamentos caducados para proceder a una disposición final adecuada y segura. Hasta el año 2019, esta campaña contó con 449 puntos de acopio de residuos en Quito, de los cuales solo 25 eran puntos de acopio para medicamentos caducados ${ }^{(14,15)}$.

A pesar de los esfuerzos por instaurar estrategias de eliminación para los medicamentos caducados y sobrantes, persisten las prácticas inadecuadas de eliminación de medicamentos que se realizan a través de la basura común, descarte por el inodoro o por grifo del fregadero tanto en zonas urbanas como rurales. Estudios recientes sugieren que los desechos farmacéuticos arrojados por estas vías generan un severo impacto medioambiental, por ello se los ha denominado contaminantes emergentes ${ }^{(16,17,18,19)}$.

En Ecuador, un trabajo de titulación de grado, realizado en el año 2017, encuestó a 110 estudiantes de ingeniería y a 330 estudiantes de medicina, y mostró que el 50\% de los encuestados reconocían tener un botiquín familiar en el hogar y que el $59 \%$ de los encuestados utilizaban de manera muy esporádica estos medicamentos, mientras que un $13 \%$ lo usaban al menos dos veces al mes ${ }^{(20)}$. Sin embargo, los autores no mencionan datos sobre los métodos de eliminación de los medicamentos caducados o sobrantes. Por otro 
lado, un estudio publicado en el mismo año, y realizado en Cuenca (la tercera ciudad más grande de Ecuador) evidenció que hasta un $70 \%$ de sus encuestados eliminan los medicamentos considerados inservibles o innecesarios junto con el resto de la basura común. Hasta un $12 \%$ afirmaron tener medicamentos caducados en su hogar y un $8 \%$ desconocía la fecha de caducidad ${ }^{(21)}$.

El presente estudio tuvo como objetivo primario identificar las prácticas de desecho más frecuentes de los medicamentos caducados y sobrantes del botiquín familiar, y como objetivo secundario caracterizar los medicamentos que forman parte del botiquín familiar (sobrantes y caducados) de los estudiantes de la carrera de medicina de la Universidad Central del Ecuador.

\section{METODOLOGÍA}

\section{Encuesta en línea}

Se realizó un estudio transversal en la ciudad de Quito, Ecuador, en la Carrera de Medicina de la Facultad de Ciencias Médicas, Universidad Central del Ecuador, entre diciembre de 2018 y enero de 2019. Se realizó una encuesta anónima en línea estructurada con preguntas relacionadas con el almacenaje y desecho de los medicamentos en los hogares. Mediante campañas en redes sociales, se invitó a participar a todos los estudiantes desde preuniversitario al décimo semestre de la carrera de Medicina. Aquellos que aceptaron participar en este estudio dieron su consentimiento para el uso de los datos para análisis.

La encuesta tenía una primera sección de datos demográficos. La segunda sección contenía siete preguntas, sobre las actitudes frente al desecho de medicamentos en el hogar. La encuesta fue desarrollada en Google Forms. Para evitar datos perdidos todas las preguntas fueron obligatorias.

Se estableció que los estudiantes que desearan participar en esta encuesta debían entregar, de manera obligatoria, sus medicamentos sobrantes de tratamientos previos o medicamentos caducados en las instalaciones de la asignatura de Farmacología. Aquellos participantes que no llenaron la encuesta o que no entregaran medicamentos que formaran parte de su botiquín familiar fueron excluidos de los análisis. El presente estudio contó con la aprobación de la coordinación de la asignatura de Farmacología como parte del proyecto integrador de saberes de la carrera de Medicina. Basado en el número de estudiantes de la carrera y asumiendo una tasa de respuesta del $50 \%$, el tamaño muestral calculado fue de 339 estudiantes con un intervalo de confianza del $95 \%$ y un margen de error del 5\% calculado en Epilnfo.

\section{Datos sobre medicamentos sobrantes o caducados recolectados}

Se creó una base de datos para caracterizar los medicamentos sobrantes y caducados recibidos (desechos farmacéuticos). En esta base de datos se detalló cantidad de medicamentos entregados por participante, número de medicamentos sobrantes, caducados y de vigencia desconocida, fecha de caducidad en caso de estar disponible, sistema de clasificación Anatomical, Therapeutic, Chemical (ATC) correspondiente, forma farmacéutica (sólida, líquida y semisólida), medicamentos que tenían la etiqueta de entrega gratuita por el sistema de salud nacional o el Instituto Ecuatoriano de Seguridad Social (IESS), y aquellos que fueron muestras médicas. Además, los medicamentos fueron categorizados como de venta libre o venta bajo prescripción acorde a lo establecido por la agencia de medicamentos de Ecuador $^{(22)}$.

Posterior al análisis, estos desechos farmacéuticos domésticos fueron entregados al programa municipal de la ciudad para la recolección de residuos domésticos peligrosos.

\section{Métodos}

Se procedió al análisis descriptivo de las siete preguntas del formulario en línea y de los desechos farmacéuticos domésticos recibidos en la asignatura de Farmacología. Los 
resultados fueron calculados y presentados en números y porcentajes.

\section{RESULTADOS}

\section{Demográficos}

Un total de 498 de estudiantes Ilenaron la encuesta y entregaron sus desechos farmacéuticos domésticos. Las características de los encuestados se detallan en la Tabla 1. La mayoría fueron mujeres (308) y estudiantes que cursaban el primer semestre de la carrera de Medicina. La media de edad fue de 20,9 años (rango 17-38). El 49,2\% de los estudiantes tenían algún familiar con patología crónica en su hogar. Entre las patologías más frecuentes se mencionó la hipertensión, diabetes mellitus tipo 2, y enfermedades tiroideas.

Tabla 1. Características de estudiantes e información de familiares ( $n=498$ ). Quito, Ecuador, 2019.

\begin{tabular}{|l|r|r|}
\hline Características & \multicolumn{2}{|c|}{ Valor } \\
\hline Estudiantes & $n$ & $\%$ \\
\hline Género & & \\
\hline Femenino & 306 & 61,5 \\
\hline Masculino & 191 & 38,3 \\
\hline No menciona & 1 & 0,2 \\
\hline Semestre & & \\
\hline Preuniversitario & 34 & 6,8 \\
\hline Primero & 159 & 31,9 \\
\hline Segundo & 64 & 12,9 \\
\hline Tercero & 12 & 2,4 \\
\hline Quinto & 59 & 11,9 \\
\hline Sexto & 51 & 10,3 \\
\hline Séptimo & 29 & 5,8 \\
\hline Octavo & 49 & 9,8 \\
\hline Noveno & 10 & 2,0 \\
\hline Décimo & 31 & 6,2 \\
\hline Estudiantes con familiares con & & \\
\hline patologías crónicas & & 50,8 \\
\hline Sin enfermedades crónicas & 253 & 49,2 \\
\hline Con enfermedades crónicas & 245 & \\
\hline & & \\
\hline
\end{tabular}

Fuente: Elaboración propia.

\section{Actitudes sobre el desecho de medicamentos caducados o sobrantes en el hogar}

Respecto a las prácticas de eliminación de los medicamentos sobrantes y/o caducados en los hogares, de los 498 encuestados un $7,2 \%$ reconoció que alguna vez sacó los medicamentos del empaque primario para depositarlos en la basura común, un 30,3\% mencionó que al menos una vez los eliminó a través del inodoro. El $83,3 \%$ de los estudiantes mencionó que los desechaba en la basura común y el $86,3 \%$ que consideraban que los medicamentos contaminaban el medio ambiente (Tabla 2).

De la totalidad, con respecto al conocimiento de contenedores municipales destinados para el desecho de medicamentos caducados, el $79,9 \% \quad(n=398)$ desconocía de la existencia de estos contenedores o de este programa municipal, únicamente $20,1 \%(n=100)$ de personas los conocían y de estos únicamente 29 estudiantes lo usaron alguna vez.

\section{Desecho de medicamentos caducados o sobrantes en el hogar}

Los 498 estudiantes entregaron 24.681 unidades de medicamentos sobrantes o caducados en formas farmacéuticas diferentes y trajeron entre 1 y 1.842 unidades. En promedio cada estudiante entregó 40 unidades (Tabla 3).

De los 498 encuestados que entregaron medicamentos, $448 \quad(89,9 \%)$ entregaron al menos una forma farmacéutica que se encontraba caducada, $163(32,7 \%)$ por lo menos un medicamento con fecha de caducidad desconocida y $171(34,3 \%)$ por lo menos un medicamento que correspondían a muestras médicas.

De las 24.681 unidades de medicamentos totales recolectados, $17.082(69,2 \%)$ unidades corresponden a medicamentos caducados, $5.578(22,6 \%)$ a medicamentos vigentes $y$ $2.021(8,1 \%)$ a medicamentos con la fecha de caducidad desconocida. De la totalidad de unidades, un $34,4 \%$ (8.484) fueron medicamentos 
Tabla 2. Encuesta de actitudes sobre el desecho de medicamentos caducados y sobrantes en el hogar ( $n=498)$. Quito, Ecuador, 2019.

\begin{tabular}{|c|c|c|c|}
\hline Preguntas & Indicadores & $n$ & $\%$ \\
\hline \multirow{5}{*}{$\begin{array}{l}\text { Indique la frecuencia con la que desechaba los } \\
\text { medicamentos sobrantes de tratamientos previos } 0 \\
\text { caducados por el inodoro. }\end{array}$} & Muy frecuentemente & 1 & 0,2 \\
\hline & Frecuentemente & 21 & 4,2 \\
\hline & Ocasionalmente & 55 & 11,1 \\
\hline & Raramente & 74 & 14,8 \\
\hline & Nunca & 347 & 69,7 \\
\hline \multirow{5}{*}{$\begin{array}{l}\text { Indique la frecuencia con la que desechaba los } \\
\text { medicamentos sobrantes de tratamientos previos o } \\
\text { caducados en la basura doméstica }\end{array}$} & Muy frecuentemente & 52 & 10,4 \\
\hline & Frecuentemente & 87 & 17,5 \\
\hline & Ocasionalmente & 151 & 30,3 \\
\hline & Raramente & 125 & 25,1 \\
\hline & Nunca & 83 & 16,7 \\
\hline \multirow{2}{*}{$\begin{array}{l}\text { ¿Alguna vez ha extraído las pastillas de los blísteres } \\
\text { de aluminio para mezclarlos con tierra y botarlos en } \\
\text { la basura doméstica? }\end{array}$} & Sí, alguna vez & 36 & 7,2 \\
\hline & No & 462 & 92,8 \\
\hline \multirow{2}{*}{$\begin{array}{l}\text { ¿Conocía que en Quito existen contenedores para } \\
\text { desecho adecuado de medicinas caducadas por } \\
\text { parte del Municipio de Quito? }\end{array}$} & Sí & 100 & 20,1 \\
\hline & No & 398 & 79,9 \\
\hline \multirow{3}{*}{$\begin{array}{l}\text { ¿Alguna vez había desechado los medicamentos } \\
\text { caducados en los contenedores del Municipio de } \\
\text { Quito? }\end{array}$} & Sí & 29 & 5,8 \\
\hline & No & 80 & 16,1 \\
\hline & Vacío & 389 & 78,1 \\
\hline \multirow{4}{*}{$\begin{array}{l}\text { ¿Con qué frecuencia había utilizado estos } \\
\text { contenedores para medicamentos caducados? }\end{array}$} & Frecuentemente & 5 & 1,0 \\
\hline & Ocasionalmente & 17 & 3,4 \\
\hline & Raramente & 14 & 2,8 \\
\hline & Nunca & 71 & 14,2 \\
\hline \multirow{3}{*}{$\begin{array}{l}\text { ¿Estaba de acuerdo con que el desecho inadecuado } \\
\text { de medicamentos tiene un efecto negativo para el } \\
\text { medio ambiente? }\end{array}$} & De acuerdo & 430 & 86,3 \\
\hline & Ni de acuerdo ni en desacuerdo & 54 & 10,8 \\
\hline & En desacuerdo & 14 & 2,9 \\
\hline
\end{tabular}

Tabla 3. Formas farmacéuticas recolectadas por semestre y fecha de caducidad ( $\mathrm{n}=24.681$ ). Quito, Ecuador, 2019.

\begin{tabular}{|l|c|c|c|c|c|}
\hline Semestre & $\begin{array}{c}\text { Total de } \\
\text { encuestados }\end{array}$ & $\begin{array}{c}\text { Unidades } \\
\text { caducadas }\end{array}$ & $\begin{array}{c}\text { Unidades vigentes } \\
\text { sin caducar }\end{array}$ & $\begin{array}{c}\text { Unidades con fechas de } \\
\text { caducidad desconocidas }\end{array}$ & Total \\
\hline Preuniversitario & 34 & 1.084 & 212 & 216 & 1.512 \\
\hline Primero & 159 & 4.995 & 1.046 & 957 & 6.998 \\
\hline Segundo & 64 & 1.275 & 227 & 91 & 1.593 \\
\hline Tercero & 12 & 272 & 157 & 86 & 515 \\
\hline Quinto & 59 & 4.463 & 2.076 & 343 & 6.882 \\
\hline Sexto & 51 & 912 & 1.122 & 135 & 2.169 \\
\hline Séptimo & 29 & 1.112 & 161 & 87 & 1.360 \\
\hline Octavo & 49 & 1.084 & 284 & 76 & 1.444 \\
\hline Noveno & 10 & 773 & 3 & 0 & 776 \\
\hline Décimo & 31 & 1.112 & 290 & 30 & 1.432 \\
\hline Total & 498 & 17.082 & 5.578 & 2.021 & 24.681 \\
\hline
\end{tabular}

Fuente: Elaboración propia. 
Tabla 4. Distribución de medicamentos recolectados $(\mathrm{N}=24,681)$, según sistema de clasificación Anatomical, Therapeutic, Chemical (ATC). Quito, Ecuador, 2019.

\begin{tabular}{|c|c|c|c|c|}
\hline \multirow[t]{2}{*}{ Sistema de clasificación ATC } & \multicolumn{2}{|c|}{ Unidades } & \multirow[t]{2}{*}{ Nombre } & \multirow[t]{2}{*}{ Cantidad } \\
\hline & $\mathrm{n}$ & $\%$ & & \\
\hline \multirow[t]{3}{*}{ A Sistema digestivo y metabolismo } & \multirow[t]{3}{*}{6.203} & \multirow[t]{3}{*}{25,1} & Metformina & 918 \\
\hline & & & Omeprazol & 483 \\
\hline & & & Multivitaminas & 437 \\
\hline \multirow[t]{3}{*}{ N Sistema nervioso } & \multirow[t]{3}{*}{4.467} & \multirow[t]{3}{*}{18,1} & Acetaminofén & 681 \\
\hline & & & Ácido acetilsalicílico & 368 \\
\hline & & & Acetaminofén + cafeína & 218 \\
\hline \multirow[t]{3}{*}{ C Sistema cardiovascular } & \multirow[t]{3}{*}{3.937} & \multirow[t]{3}{*}{15,9} & Espironolactona & 682 \\
\hline & & & Enalapril & 445 \\
\hline & & & Simvastatina & 374 \\
\hline \multirow[t]{3}{*}{ J Antiinfecciosos en general para uso sistémico } & \multirow[t]{3}{*}{2.513} & \multirow[t]{3}{*}{10,2} & Amoxicilina & 396 \\
\hline & & & Ciprofloxacina & 224 \\
\hline & & & Amoxicilina + ácido clavulánico & 221 \\
\hline \multirow[t]{3}{*}{ M Sistema musculo esquelético } & \multirow[t]{3}{*}{1.978} & \multirow[t]{3}{*}{8,0} & Ibuprofeno & 614 \\
\hline & & & Diclofenaco & 306 \\
\hline & & & Naproxeno & 207 \\
\hline \multirow[t]{3}{*}{ R Sistema respiratorio } & \multirow[t]{3}{*}{1.244} & \multirow[t]{3}{*}{5,1} & Loratadina & 345 \\
\hline & & & Dimenhidrinato & 104 \\
\hline & & & Tiotropio & 90 \\
\hline \multirow[t]{3}{*}{ B Sangre y órganos hematopoyéticos } & \multirow[t]{3}{*}{1.161} & \multirow[t]{3}{*}{4,7} & Ácido fólico & 490 \\
\hline & & & Clopidogrel & 191 \\
\hline & & & Sulfato de hierro & 151 \\
\hline \multirow[t]{3}{*}{ G Aparato genitourinario y hormonas sexuales } & 744 & 3,1 & Levonorgestrel + etinilestradiol & 251 \\
\hline & & & Tamsulosina & 200 \\
\hline & & & Isoxuprina & 70 \\
\hline L Agentes antineoplásicos e inmunomoduladores & 570 & 2,3 & Ácido micofenólico & 570 \\
\hline H Preparados hormonales sistémicos, excluyendo & 317 & 1,3 & Levotiroxina & 182 \\
\hline & & & Prednisona & 120 \\
\hline & & & Metilprednisolona & 5 \\
\hline P Productos antiparasitarios, insecticidas y repelentes & 274 & 1,1 & Arteméter + lumefantrina & 79 \\
\hline & & & Nitazoxanida & 47 \\
\hline & & & Quinina & 34 \\
\hline D Medicamentos dermatológicos & 234 & 0,9 & Nitrato de miconazol + tinidazol & 26 \\
\hline & & & Terbinafina & 25 \\
\hline & & & Betametasona & 13 \\
\hline SÓrganos de los sentidos & 33 & 0,1 & $\begin{array}{l}\text { Lágrimas artificiales y otras } \\
\text { preparaciones indiferentes }\end{array}$ & 5 \\
\hline & & & Sulfacetamida & 3 \\
\hline Varios & 43 & 0,2 & & \\
\hline Natural & 44 & 0,2 & & \\
\hline Desconocido & 919 & 3,7 & & \\
\hline
\end{tabular}


que tuvieron etiqueta de entrega gratuita por el Ministerio de Salud Pública o Instituto Ecuatoriano de Seguridad Social, el 14,8\% (3.651) fueron identificados como muestras médicas y un $50,8 \%$ (12.546) no tuvieron ninguna identificación de entrega gratuita.

Acorde a la clasificación ATC, la mayoría de los medicamentos correspondían al grupo A (sistema digestivo y metabolismo), siendo la metformina el principio activo más frecuente con 918 unidades, seguido del omeprazol y los multivitamínicos. El grupo $\mathrm{N}$ (sistema nervioso) fue el segundo más frecuente, con el acetaminofén como el principio activo más frecuente. El tercer grupo más frecuente fue el cardiovascular y el cuarto grupo fue el J (antiinfecciosos en general para uso sistémico). Todos los grupos ATC y sus respectivos números de frecuencia se describen en la Tabla 4.

Del total de unidades, 23.497 (95,2\%) fueron formas farmacéuticas sólidas, de las cuales las tabletas fueron las formas farmacéuticas más frecuentes. Los principios activos más frecuentes en tabletas fueron: ácido acetilsalicílico, seguido por metformina e ibuprofeno. Un 3,6\% (887) fueron formas farmacéuticas líquidas y un 1,2\% (297) fueron formas farmacéuticas semisólidas.

Del total de unidades recolectadas, 7.198 $(29,2 \%)$ fueron medicamentos catalogados como de venta libre-over the counter (OTC)por la agencia nacional reguladora de medicamentos de Ecuador. Un total de 16.961 $(68,7 \%)$ fueron catalogados como medicamentos bajo prescripción médica y $522(2,1 \%)$ no se pudo establecer puesto que se desconocía el nombre del medicamento o el principio activo. En aquellos medicamentos catalogados como de venta bajo prescripción médica, los más frecuentes fueron la metformina con 707 unidades $(2,9 \%)$, seguido por el ácido micofenólico con 570 (2,3\%), el ácido fólico con 490 $(1,9 \%)$ y la simvastatina con $414(1,7 \%)$.

De las 17.082 unidades caducadas, el $32,3 \%$ tuvieron la etiqueta de entrega gratuita por parte del Ministerio de Salud Pública (1.655) o por parte del Instituto Ecuatoriano de Seguridad Social (3.870), siendo los más frecuentes en orden descendente: espironolactona con 425 unidades, metformina con 400, y ácido acetilsalić́lico con 311. Un $16,9 \%$ (2.891) del total de caducados correspondió a muestras médicas. El $50,7 \%$ restante de las unidades caducadas (8.666) correspondería a compra privada doméstica.

De las unidades con fecha de caducidad desconocida (2.021 unidades), se pudo identificar que el principio activo más frecuente fue el acetaminofén con 126 unidades.

\section{DISCUSIÓN Y CONCLUSIONES}

En este estudio, un $83,3 \%$ de los encuestados admitió haber desechado alguna vez los medicamentos íntegros en la basura común, un 7,2\% reconoció sacar las unidades de sus blísteres o empaques primarios para desecharlos en la basura común y hasta un $30,3 \%$ los desechó al inodoro o por el grifo del fregadero al menos una vez. Además, el $89,9 \%$ de los encuestados tenía en su botiquín familiar por lo menos un medicamento caducado, y hasta un $32,7 \%$ tuvieron en su botiquín familiar medicamentos en los que la fecha de caducidad era desconocida debido a un incorrecto almacenamiento en el hogar.

Las prácticas de desecho de medicamentos se encuentran influenciadas por la conciencia ambiental, actitudes sociales y culturales. Estudios estiman que más de la mitad de los medicamentos no utilizados, se desecha de manera inadecuada. Un estudio realizado en Costa Rica señaló que el $84 \%$ de las personas encuestadas mencionó que la principal forma de desecho de los medicamentos en el ámbito doméstico era eliminarlos junto con la basura común y el 17\% los arrojaban al inodoro ${ }^{(23)}$. En Colombia se evidenciaron resultados similares; de hecho, en el caso de medicamentos caducados, el $64 \%$ los eliminaba junto con la basura común mientras que, en el caso de medicamentos sobrantes, un $26 \%$ los eliminaba en la basura común. Los autores colombianos además señalaron que, independientemente del estado de caducidad, solo un $9 \%$ los 
eliminaba por el inodoro ${ }^{(24)}$. Estos resultados de la región latinoamericana son similares a los observados en la región de Asia ${ }^{(25,26)}$.

En los países de Latinoamérica existen varias estrategias encaminadas a mejorar la disposición final de los medicamentos caducados y sobrantes en el hogar ${ }^{(16,24,27,28,29)}$. Sin embargo, estas estrategias todavía se encuentran en fases iniciales de implementación por lo que, a pesar de los esfuerzos, sigue siendo un problema con impacto ambiental y en la salud pública.

Es importante señalar que, del total de medicamentos entregados, hasta un 50,7\% fueron medicamentos obtenidos por compra privada, esto se logró estimar ya que los sistemas de entrega gratuita llevan impreso la leyenda de gratuidad en los envases primarios y secundarios. Este hallazgo nos permite considerar la hipótesis de que existe un alto gasto en la compra de medicamentos en las familias ecuatorianas, en especial cuando se trata de patologías crónicas. Además, otra consideración a tomar en cuenta es la probable falta de control estricto en la venta de medicamentos bajo prescripción médica en el país. En Ecuador, el Art. 153 de la Ley Orgánica de la Salud, ley máxima que rige el sistema sanitario en el país, establece que se requiere receta médica para la compra de todos los medicamentos, excepto aquellos catalogados como de venta libre ${ }^{(30)}$. Existen dos tipos de recetas médicas: la de prescripción de medicamentos, de uso y consumo humano; y la receta especial, únicamente para medicamentos que contienen sustancias catalogadas sujetas a fiscalización (estupefacientes y psicotrópicas) $)^{(31)}$.

Sin embargo, a pesar de la existencia de esta ley, el $68,7 \%$ de los medicamentos que se observaron como parte de los botiquines familiares fueron de venta bajo prescripción médica, en especial, aquellos utilizados en patologías crónicas tales como diabetes y dislipidemia, e incluso medicamentos de uso más específico (ácido micofenólico). Este hallazgo podría estar justificado por la presencia de dos o más patologías crónicas en las familias de los estudiantes; sin embargo, este dato contrasta con otros estudios en los que se observa con mayor frecuencia en los botiquines familiares la presencia de analgésicos y antiinflamatorios (medicamentos de venta libre) ${ }^{(2,32)}$.

Además, en nuestro estudio se evidenció la existencia de un alto grado de almacenamiento de medicamentos en botiquines familiares, ya que cada estudiante en promedio entregó aproximadamente 40 unidades de medicamentos caducados o sobrantes, esto es preocupante porque tal cantidad de medicamentos podría predisponer a una automedicación riesgosa para el individuo, problemática que es considerada de relevancia internacional ${ }^{(33,34,35)}$. En este contexto, es importante recalcar la alta presencia de antibióticos en los botiquines familiares, lo que podría favorecer al uso indiscriminado de los antibióticos por automedicación y así contribuir además a la resistencia antimicrobiana como se han analizado Torres et al. en un metaanálisis ${ }^{(36)}$.

Otro hallazgo relevante fue la presencia de un $32,3 \%$ de medicamentos caducados entregados por los sistemas de salud gratuita del país, en los que se repite el hallazgo de medicamentos, como la metformina, utilizados para patologías crónicas. Esto podría señalar la necesidad de implementar un sistema de reciclaje de medicamentos por cambio de tratamiento en las instancias del Ministerio de Salud y redes anexas, puesto que el almacenaje en casa y posterior caducidad representan, además de un riesgo para la familia, un alto gasto para el sistema de salud.

El desecho adecuado y la disposición final de los medicamentos caducados y sobrantes en el hogar representan una problemática social, que obedece principalmente a una falta de estrategias intersectoriales locales y nacionales para el desecho seguro de estos medicamentos. Es necesario un compromiso de todos los actores involucrados en esta problemática, instituciones de cuidado medio ambiental, instituciones de salud pública, industria farmacéutica, sociedades científicas y ciudadanos para desarrollar programas de educación y, al mismo tiempo, implementar estrategias consolidadas que permitan atacar este problema lo más pronto posible. 
En diferentes países europeos existen estrategias consolidadas para controlar y disminuir el impacto negativo de las prácticas inadecuadas de desecho de los medicamentos. Estas estrategias son el resultado de esfuerzos multisectoriales. El punto inicial de estas estrategias son las farmacias comunitarias ya que, en estos establecimientos de salud, existe contenedores para recibir medicamentos caducados, y además en otros establecimientos parte del sistema sanitario existe el reciclaje de medicación por cambio de tratamiento farmacológico, especialmente en patologías crónicas. Es importante mencionar que estas estrategias resultan exitosas ya que además existen políticas de medicamentos que contribuyen a un uso racional de medicamentos, controlando la compra y venta sin receta médica ${ }^{(11)}$.

En conclusión, este estudio evidencia la urgente necesidad de desarrollar políticas intersectoriales para la concientización e implementación de un desecho seguro de medicamentos caducados o sobrantes de origen doméstico. En la capital del país existe un plan de desecho de medicamentos caducados, los análisis de este proyecto pueden ayudar a entender la problemática en el contexto local para plantear soluciones que potencialmente se extiendan a todo el país. Acorde a nuestra revisión, además del estudio realizado en la ciudad de Cuenta, no existen otros estudios publicados sobre este tema en Ecuador, por lo que es necesario más estudios para conocer las prácticas de desecho en sectores rurales y en diversas poblaciones, puesto que es una limitación de este estudio la población seleccionada de estudiantes de Medicina. Además, se requieren estudios enfocados en el análisis del impacto medioambiental y las potenciales consecuencias en la salud del desecho doméstico inadecuado de medicamentos. Por otro lado, los hallazgos aquí presentados señalan la necesidad de un control sobre la venta de medicamentos en las farmacias, dado que la acumulación de medicamentos puede llevar a una automedicación riesgosa en los miembros del hogar, así como también ser fuente de potenciales intoxicaciones.

\section{FINANCIAMIENTO}

La investigación que dio origen a este artículo no recibió financiamiento.

\section{CONFLICTOS DE INTERESES}

El autor y la autora de este trabajo declaran que, si bien estudian y trabajan en la misma institución que facilitó los datos analizados en este estudio, se tomaron todos los recaudos necesarios para asegurar que este vínculo no representara conflicto de intereses ni condicionara el tipo de análisis y las conclusiones a las que se arriban en este artículo.

\section{AGRADECIMIENTO}

Este trabajo fue realizado en el contexto de proyecto integrador de saberes de la Asignatura de Farmacología, implementado por la Universidad Central del Ecuador. Un sincero agradecimiento a todos los estudiantes que colaboraron con el proyecto y especialmente a aquellos que ayudaron a la recolección, clasificación y correcto desecho de los medicamentos: Andrade Katherine, Chininín Daniel, Escobar Dayanna, Calderón Juan-Mateo, Méndez Erik, Villota Camila, Paca Vladimir, Villagómez Alexandra, Vallejo Carol, Yambay Cristina, Cepeda Fernando, Álvaro Edgar y Orozco Diego.

\section{REFERENCIAS BIBLIOGRÁFICAS}

1. Cruz E, Larrubia O, Pérez M, Taravilla M. El botiquin casero [Internet]. 2011 [citado 1 feb 2019]. Disponible en: https://tinyurl.com/2yejr9ma.

2. Solá M, Garrido E, Úbeda I, Morin V, Sancho R, Fabrellas $\mathrm{N}$. Analisis de los botiquines domésticos: una experiencia de aprendizaje significativa para los estudiantes de enfermería. Texto \& Contexto Enfermagem. 2016;25(1):1-7.

3. Vellinga A, Cormican S, Driscoll J, Furey M, O'Sullivan $M$, Cormican M. Public practice regarding disposal of unused medicines in Ireland. Science of the Total Environment. 2014;478:98-102.

4. Quadra GR, Silva PSA, Paranaíba JR, Josué IIP, Souza H, Costa R, et al. Investigation of medicines consumption and disposal in Brazil: A study case in a developing country. Science of the Total Environment. 2019;671:505-509.

5. Quadra GR, Souza HO, Costa RS, Fernandez MAS. Do pharmaceuticals reach and affect the aquatic ecosystems in Brazil? A critical review of current studies in a developing country. Environmental Science and Pollution Research. 2017;24(2):1200-1218. 
6. Indu R, Adhikari A, Maisnam I, Basak P, Sur TK, Das AK. Polypharmacy and comorbidity status in the treatment of type 2 diabetic patients attending a tertiary care hospital: An observational and questionnaire-based study. Perspective in Clinical Research. 2018;9(3):139144.

7. Arias JL, Ruiz MA, Gallardo V. El futuro farmacéutico y el botiquín familiar: Aprendiendo a realizar educación sanitaria. Ars Pharmaceutica. 2009;50(3):118-123.

8. Prüss A, Giroult E, Rushbrook P. Safe management of wastes from health-care activities [Internet]. 1999 [citado 30 abr 2021]. Disponible en: https://tinyurl. com/2cc9dju7.

9. Societies RC, Federation IP. Safe disposal of unwanted pharmaceuticals in and after emergencies. Revista Panamericana de Salud Pública. 2000;7(3):205-208.

10. Carvajal F, Mora J. Medicamentos no utilizables: problemática y medidas pertinentes para su disposición final. Revista Médica de la Universidad de Costa Rica. 2016;10(1):27-36

11. Health Care without harm. Unused pharmaceuticals where do they end up? [Internet]. 2013 [citado $30 \mathrm{abr}$ 2021]. Disponible en: https://tinyurl.com/yut7ynpm.

12. Medicamentos y Medio Ambiente. Sistema Integrado de Gestión de Residuos de medicamentos de origen domiciliario y sus Envases [Internet]. 2020 [citado 30 abr 2020]. Disponible en: https://www.sigre.es.

13. INFAC. Farmacontaminación: impacto ambiental de los medicamentos [Internet]. 2016;24(10):59-64 [citado 8 mar 2019]. Disponible en: https://tinyurl. $\mathrm{com} / 55 \mathrm{bwcb} 4 \mathrm{f}$.

14. La Hora. En Quito existen 449 puntos donde reciclar diferentes tipos de desechos [Internet]. 2019 [citado 30 abr 2021]. Disponible en: https://tinyurl.com/bz3rktnm.

15. EMASEO. Quito a reciclar [Internet]. 2020 [citado 30 abr 2021]. Disponible en: https://tinyurl.com/netk4kcx.

16. Jáuregui Medina C, Ramos Ramírez LC, Figueroa Morales JR, Medina Carrillo RE, Rodríguez Castañeda I, Padilla Noriega R. México hacia una cultura sobre la disposición final de medicamentos caducados. Revista Fuente Nueva Época. 2015;20(6):24-31.

17. Moreno-Ortiz VC, Martínez-Núñez JM, KravzovJinich J, Pérez-Hernández LA, Moreno-Bonett C, Altagracia-Martínez M. Los medicamentos de receta de origen sintético y su impacto en el medio ambiente. Revista Mexicana de Ciencias Farmacéuticas. 2013;44(4):17-29

18. Berrocal RA, Rojas MA, Santana Cl. Disposición final de medicamentos por universitario. Revista Electrónica de Portales Médicos. 2019;14(22):518.

19. Williams E, Brooks B. Human Health risk assessment for pharmaceuticals in the environment: existing practice, uncertainty, and future directions. En: Brooks BW, Huggett DB, eds. Human pharmaceuticals in the environment current and future perspectives, emerging topics in ecotoxicology. New York: Springer Science; 2012. p. 167-224.

20. Ortiz Simbaña JA. Análisis de las características del botiquín familiar de estudiantes del área de la salud en la ESPOCH, como aporte al control de la automedicación y la educación sanitaria [Tesis de grado]. Riobamba: Escuela Superior Politécnica de Chimborazo; 2017.

21. Cobos F, Cisneros J. Manejo de desechos farmacéuticos de origen doméstico. Estudio en la Parroquia Gil Ramírez Dávalos en Cuenca-Ecuador. Revista de la Facultad de Ciencias Químicas. 2017;(17):29-40.

22. Agencia Nacional de Regulación Control y Vigilancia Sanitaria. Base de Registros Emitidos [Internet]. 2021 [citado 30 abr 2021]. Disponible en: https://tinyurl. com/2hch24dn.

23. Jiménez HLG, Blanco Sáenz R. Medicamentos subutilizados en el ámbito comunitario, área salud coronado, Costa Rica. Revista Costarricense de Salud Pública. 2006;15(28):50-61.

24. Quijano-Prieto DM, Orozco-Díaz JG, Holguín-Hernández $\mathrm{E}$. Conocimientos y prácticas de pacientes sobre disposición de medicamentos no consumidos: Aproximación a la ecofarmacovigilancia. Revista de Salud Publica. 2016;18(1):61-71.

25. Azad MAK, Ansary MRH, Akhter MA, Al-Mamun SMM, Uddin M, Rahman MM. Disposal practice for unused medications among the students of the international islamic university Malaysia. Journal of Applied Pharmaceutical Science. 2012;2(7):101-106.

26. Jha N, Shankar PR, Palaian S. Knowledge and practice on ecopharmacovigilance and medicine storage amongst medical and dental students in Lalitpur, Nepal. Risk Management and Healthcare Policy. 2021;14:793802.

27. Graciani FS, Ferreira GLBV. Impacto ambiental de los medicamentos y su regulación en Brasil. Revista Cubana de Salud Pública. 2014;40(2):268-273.

28. Republica de Costa Rica. Reglamento para la disposición final de medicamentos, materias primas, y sus residuos No 36039-S [Internet]. 2010 [citado 30 abr 2021]. Disponible en: https://tinyurl.com/9f4m2vrx.

29. Dongo V. Ley No 29459-Ley de los Productos Farmacéuticos, Dispositivos Médicos y Productos Sanitarios. Revista Peruana de Medicina Experimental y Salud Pública. 2009;26(4):517-546.

30. Ecuador. Ley Organica de Salud: Ley 67 [Internet]. 2015 [citado 30 abr 2021]. Disponible en: https://tinyurl. com/6je3vx29.

31. Ministerio de Salud Pública. Reglamento para establecer el contenido y requisitos de la receta médica y control de la prescripción, dispensación y expendio para medicamentos de uso y consumo humano: registro oficial No. 127 [Internet]. 2020 [citado 30 abr 2021]. Disponible en: https://tinyurl.com/5mhrvt8z. 
32. De Bolle L, Mehuys E, Adriaens E, Remon J-P, Van Bortel L, Christiaens T. Home Medication Cabinets and Self-Medication: A Source of Potential Health Threats? Annals Pharmacotherapy. 2008;42(4):572-579.

33. Tesfamariam S, Anand IS, Kaleab G, Berhane S, Woldai B, Habte E, et al. Self-medication with over the counter drugs, prevalence of risky practice and its associated factors in pharmacy outlets of Asmara, Eritrea. BMC Public Health. 2019;19(1):1-10.

34. Vacas Rodilla E, Castellà Dagà I, Sánchez Giralt M, Pujol Algué A, Pallarés Comalada MC, Balagué Corbera
M. Automedicación y ancianos. La realidad de un botiquín casero. Atención Primaria. 2009;41(5):269-274.

35. Baos VV. Estrategias para reducir los riesgos de la automedicación. Información Terapéutica del Sistema Nacional de Salud. 2000;24(6):147-152.

36. Torres NF, Chibi B, Kuupiel D, Solomon VP, Mashamba-Thompson TP, Middleton LE. The use of non-prescribed antibiotics; prevalence estimates in lowand-middle-income countries: A systematic review and meta-analysis. Archives of Public Health. 2021;79(2).

\section{FORMA DE CITAR}

Calderón JM, Tarapués M. Medicamentos sobrantes y caducados en el hogar ¿su almacenaje y desecho representan un problema de salud pública? Salud Colectiva. 2021;17:e3599. doi: 10.18294/sc.2021.3599.

Recibido: 12 may 2021 | Versión final: 24 ago 2021 | Aprobado: 9 sep 2021 | Publicado en línea: 13 oct 2021

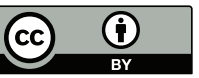

Esta obra está bajo una licencia Creative Commons Atribución 4.0 Internacional (CC BY 4.0). Atribución - Se debe dar crédito de manera adecuada, brindar un enlace a la licencia, e indicar si se han realizado cambios. Puede hacerlo en cualquier forma razonable, pero no de forma tal que sugiera que usted o su uso tienen el apoyo de la licenciante. Sin restricciones adicionales - No se pueden aplicar términos legales ni medidas tecnológicas que restrinjan legalmente a otras personas a hacer cualquier uso permitido por la licencia. 